\title{
Filigrane
}

Écoutes psychanalytiques

\section{Clinique des adolescents en conflit avec la loi : contributions de la psychanalyse au travail de la demande}

\author{
Laís Macêdo Vilas Boas, Deise Matos do Amparo, Sandra Francesca Conte de \\ Almeida et Katia Cristina Tarouquella R. Brasil
}

Volume 24, numéro 2, automne 2015

Le devenir de la psychanalyse. Que nous disent les pratiques institutionnelles?

URI : https://id.erudit.org/iderudit/1036531ar

DOI : https://doi.org/10.7202/1036531ar

Aller au sommaire du numéro

\section{Éditeur(s)}

Revue Santé mentale au Québec

ISSN

1192-1412 (imprimé)

1911-4656 (numérique)

Découvrir la revue

Citer cet article

Vilas Boas, L. M., do Amparo, D. M., Conte de Almeida, S. F. \& Tarouquella R. Brasil, K. C. (2015). Clinique des adolescents en conflit avec la loi : contributions de la psychanalyse au travail de la demande. Filigrane, 24(2), 57-77.

https://doi.org/10.7202/1036531ar

\section{Résumé de l'article}

Cet article aborde la question complexe de la demande et du transfert dans la prise en charge clinique des adolescents en conflit avec la loi, où l'agir violent devient une modalité d'expression. Cette clinique impose d'emblée une complication pour nos pratiques : celle de travailler la demande initiale et en faire une réelle démarche d'aide, car ces jeunes trouvent dans l'acte le principal recours pour faire face à l'angoisse. À partir d'une vignette clinique, nous discutons certains aspects de la problématique en nous appuyant sur l'approche théorico-clinique de la psychanalyse. L'angoisse de l'adolescent en analyse a été prise comme un signe de demande d'aide face à la détresse non soutenable due au manque de structure de l'appareil psychique. Nous considérons alors que l'analyse a produit des effets en termes d'élaboration psychique, car des associations et des liens ont été effectués au moyen de récits chargés d'affects, liés à l'histoire de vie de l'adolescent. Le maniement du rapport transférentiel a été fondamental et a contribué au passage d'une demande sous mandat judiciaire à une demande d'analyse, et ce même dans un contexte difficile pour l'établissement d'un cadre de travail. C'est dans l'impasse de l'acte de symbolisation que la clinique psychanalytique est à même de montrer son pouvoir, au moment où elle convoque le sujet à utiliser la parole et s'impliquer dans son acte, dans son discours et dans son désir. 


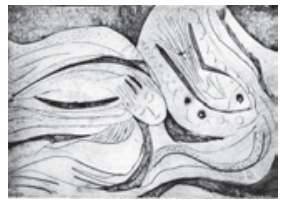

\title{
Clinique des adolescents en conflit avec la loi: contributions de la psychanalyse au travail de la demande ${ }^{1}$
}

\author{
Laís Macêdo Vilas Boas, Deise \\ Matos do Amparo, Sandra \\ Francesca Conte de Almeida et \\ Katia Cristina Tarouquella R. Brasil
}

\begin{abstract}
Cet article aborde la question complexe de la demande et du transfert dans la prise en charge clinique des adolescents en conflit avec la loi, où l'agir violent devient une modalité d'expression. Cette clinique impose d'emblée une complication pour nos pratiques: celle de travailler la demande initiale et en faire une réelle démarche d'aide, car ces jeunes trouvent dans l'acte le principal recours pour faire face à l'angoisse. À partir d'une vignette clinique, nous discutons certains aspects de la problématique en nous appuyant sur l'approche théorico-clinique de la psychanalyse. L'angoisse de l'adolescent en analyse a été prise comme un signe de demande d'aide face à la détresse non soutenable due au manque de structure de l'appareil psychique. Nous considérons alors que l'analyse a produit des effets en termes d'élaboration psychique, car des associations et des liens ont été effectués au moyen de récits chargés d'affects, liés à l'histoire de vie de l'adolescent. Le maniement du rapport transférentiel a été fondamental et a contribué au passage d'une demande sous mandat judiciaire à une demande d'analyse, et ce même dans un contexte difficile pour l'établissement d'un cadre de travail. C'est dans l'impasse de l'acte de symbolisation que la clinique psychanalytique est à même de montrer son pouvoir, au moment où elle convoque le sujet à utiliser la parole et s'impliquer dans son acte, dans son discours et dans son désir.
\end{abstract}

\section{Demande d'analyse ou analyse par mandat?}

our Freud (1926), ce qui s'impose comme essentiel au début d'une analyse est de savoir si le symptôme du sujet peut être travaillé par l'intermédiaire du processus analytique. Le symptôme névrotique, qui traduit un conflit et provoque une souffrance psychique, est celui qui a déterminé la 
construction du dispositif analytique. Freud (1913), dans le texte Le début $d u$ traitement, propose qu'avant d'engager une analyse, il faut que le patient soit amené à s'installer dans la dynamique de l'associativité par le biais des rencontres préalables pendant quelques semaines. Lors de ces rencontres, il serait possible de mettre en question l'efficacité de la méthode psychanalytique pour ce sujet.

En 1905 [1904], dans le texte De la psychothérapie, Freud affirme clairement que la psychothérapie n'est pas applicable à des personnes qui s'y sont soumises uniquement par l'autorité des proches et non en raison de leur souffrance. Cependant, Freud ne prend pas cette prémisse à la lettre car, quelques années plus tard, en 1920, il engage le traitement d'une jeune homosexuelle dont la demande provient, originellement, de sa famille. Toutefois, cette question représente sans doute un nœud gordien pour la théorie et la clinique psychanalytique: comment transformer une demande, que ce soit par mandat d'une autorité ou même par une demande d'aide, en une demande d'analyse?

L'élaboration de la demande d'analyse prend effet à partir du moment où le sujet se reconnaît dans sa souffrance, c'est-à-dire, où il reconnaît sa participation au symptôme et est capable de faire une énigme de sa plainte, tout en construisant une toile de réponses au long de l'analyse. Ce qui signifie que l'implication du sujet dans son symptôme constitue, comme le rappelle Freud (1914), le premier pas du traitement psychanalytique: «tout d'abord et avant tout, le début du traitement en soi provoque en changement dans l'attitude consciente du patient par rapport à sa maladie» (p. 167). Lacan (1958) introduit le concept de rectification pour désigner, justement, comment le travail de l'analyste va consister à ouvrir des voies d'appropriation subjective, surtout en début de traitement. L'analyste doit faire en sorte que l'analysant s'implique dans ce qui apparaissait jusqu'alors comme des plaintes initiales posées comme des revendications. Dans cette perspective, il est fondamental que l'analyste puisse ouvrir une brèche vers l'inconscient afin que l'analysant se rende compte de la singularité de son désir, en jeu dans l'analyse. Ainsi, l'écoute de l'analyste et ses interventions (rectifications et non pas proprement des interprétations) ont pour but d'instaurer un moment où le sujet peut mettre en question les motifs qui l'ont amené à l'analyse, en abandonnant une position simplement plaintive, pour entrevoir son symptôme comme articulé à sa subjectivité ou, en d'autres termes, au désir inconscient qui l'habite et qui régit son rapport à l'Autre. 
Si Freud suggérait qu'une analyse ne puisse se soutenir que si la demande advient du sujet lui-même, il nous apparait aujourd'hui plausible que l'analyste puisse accueillir une demande d'aide, même sous mandat d'une autorité extérieure. Il ne s'agit plus seulement de savoir s'il est possible de réaliser un travail analytique à partir d'une certaine demande, mais d'apporter des rectifications, dans les entretiens initiaux, pour que le sujet puisse s'impliquer subjectivement dans la demande, c'est-à-dire, pour que soit créé un désir d'analyse, un symptôme analytique, avec la contribution de l'analyste. Pour cette raison, parmi d'autres, la clinique avec des adolescents en conflit avec la loi acquiert un intérêt particulier.

La psychothérapie psychanalytique avec ces adolescents ne trouve pas toujours le support de la demande des proches, ceux-ci pouvant également être absents de leur vie pour plusieurs raisons. Maintes fois leurs vies furent marquées par la transgression, ce qui provoque dans leur famille des sentiments de détresse et d'impuissance, voire de désespoir envers ces jeunes qui ne peuvent donc compter sur leur soutien affectif. Ce qui émerge alors comme demande chez ces adolescents nous vient de manière indirecte, par l'intermédiaire d'un mandat porté par ceux qui se «substituent » aux parents: l'autorité judiciaire et/ou les éducateurs qui vont s'impliquer en rapport aux normes, à la morale. En placement ou en semi-liberté, l'adolescent est toujours confronté à des professionnels représentant l'État. Néanmoins, le travail psychanalytique est d'une autre nature et ne se confond pas avec l'image de l'autorité morale et des bons usages. Quand est proposée à ces adolescents une écoute psychanalytique, quelques concepts théorico-cliniques sont d'emblée mis en jeu, tels que la demande du sujet, le lieu du transfert, le cadre et les ambivalences institutionnelles.

Selon Arnaux (2003), la pratique de la psychanalyse en dehors du cadre psychanalytique classique peut entrainer des risques, comme la menace au processus associatif et un cadre défaillant, provoquant la difficulté à soutenir le processus psychothérapique. Roussillon (1999) souligne, en référence aux problématiques narcissico-identitaires, que dans ces situations, il faut mettre à disposition un cadre qui tienne compte de la problématique clinique. Cette proposition nous mène à la «clinique des extrêmes» et nous convoque à une nouvelle posture face à un nouvel objet d'investigation et à la réorganisation du cadre psychothérapique, car les sujets transgressent les limites et construisent le transfert de façon particulière. Ainsi, les pathologies liées à l'agir constituent un défi pour le clinicien, puisque les symptômes se situent massivement en dehors de la scène clinique et ces sujets 
se positionnent de façon projective par rapport au clinicien et à l'équipe d'accueil. Le passage à l'acte, la force et la violence s'orientent vers l'Autre et le langage n'a pas exactement le statut de communication intersubjective, les rapports entraînant en permanence le risque de la violence et de la destruction, ce qui met en évidence une problématique limite. Dans ce contexte clinique, comment construire une demande d'analyse?

On considère que cette clinique requiert un travail de désidentification par rapport à la demande sociale et institutionnelle afin d'autoriser la quête de la demande d'aide de l'adolescent. L'analyste doit tenir compte et s'occuper du problème énoncé par la société, représenté par l'institution d'accueil de l'adolescent, mais il est essentiel que l'analysant construise sa propre version, c'est-à-dire une question qui lui fasse énigme et dont l'inscription sera de l'ordre de la parole et non de l'agir.

En effet, l'excessive proximité de l'adolescent avec le discours social pourra compliquer la situation. En fait, plus il affirme avoir besoin de la morale sociale et de s'y conformer, plus il pourra se retrouver à distance de lui-même en présentant une résistance pour parler, pour s'interroger à propos du sens de sa délinquance. Le psychanalyste sait bien que pour le sujet, la valeur de la délinquance est différente de celle imposée par le social. Il faut alors aider l'adolescent à formuler le sens de ce comportement.

Parallèlement à la transformation d'une demande d'aide en demande d'analyse, dans le cas d'adolescents placés et privés de liberté, il est important de penser le transfert institutionnel, constitué par les multiples relations dans l'espace groupal institutionnel, tout en essayant d'éviter l'intrusion de l'institution, dans l'espace du setting analytique, si l'on considère que les murs de l'espace analytique sont les mêmes que ceux de l'institution de placement. Comment faire, alors, pour que l'analyste ne soit pas associé à l'institution et à ses murs? Comment peut-il proposer à l'adolescent un espace créatif d'élaboration et de symbolisation, même si cet espace se situe intra-muros? Il nous semble opportun de citer ici la conception de cadre proposée par Roussillon (1995). L'auteur rappelle que le cadre est un espace circonscrit, imposant une limite et une bordure, c'est-à-dire qu'il s'agit à la fois d'un cadre et d'un support. Pour la psychanalyse, cette conception acquiert un aspect dynamique pouvant assumer différentes fonctions pour différents patients. Cela fonctionne comme une scène ou une toile marquée par l'impression de chaque sujet et pouvant être repensée par l'analyste à chaque cas, selon l'histoire singulière et l'économie pulsionnelle du sujet à un certain moment. Roussillon ajoute que le cadre articule différents 
processus, tels que la matérialisation, l'abstraction, l'immobilisation et la métaphorisation. Ces processus se déploient dans une dynamique permettant une lecture économique du symptôme mais aussi, suscitant un travail de réobjectalisation propre à réinscrire le sujet dans la représentation de ses conflits avec ses premiers objets.

Cette conception est essentielle pour comprendre que le travail de l'analyste ne se limite pas seulement au champ de la parole, mais qu' il tient compte également de l'environnement de l'accueil et du corps même de l'analyste, comme une toile pour le transfert de l'analysant et comme un véhicule pour les interventions de l'analyste. De ce point de vue, comment penser un cadre qui signale des limites, qui fasse bord et qui contribue à la symbolisation d'adolescents dont la relation au monde est traversée, de façon privilégiée, par la transgression des limites?

La délinquance rend compte d'un conflit du sujet à l'égard de la loi et de la morale sociale. La psychanalyse parle du rôle de la morale dans l'apaisement des pulsions. Le conflit procède de deux flux — d'une part, le désir de caractère inconscient et, d'autre part, l'exigence civilisatrice, le refoulement et la morale. Le travail de transformer la demande en énigme exige l'accueil du désir, l'accueil de l'adolescent comme un sujet de désir reconnu au-delà de la place du délinquant. Un autre objectif de ce moment initial du travail concerne l'opération du transfert ou en d'autres mots, «l'accrochage» de l'analysant au processus analytique et à la personne de l'analyste. Lacan (1958b) récupère la valeur de ce moment logique précédant l'expérience analytique proprement dite, en le désignant comme entretiens préliminaires.

Le rapport entre transfert et demande est intime, car c'est au moyen du transfert que survient la construction da la demande analytique. Ce mouvement permet que «la plainte se transforme en demande adressée à cet analyste et que le symptôme passe du statut de réponse au statut de question pour le sujet, pour que celui-ci soit encouragé à le déchiffrer » (Quinet, 2002, p. 16). L'analyste crée les conditions qui incitent le sujet à se déchiffrer, ce qui permet que la demande soit adressée à l'analyste par le transfert.

Les entretiens préliminaires, outre les questions de la demande et du transfert, permettent à l'analyste de prendre position face au maniement du transfert, c'est-à-dire d'assumer la place d'analyste par rapport à un analysant en particulier. Une première question, très importante, concerne le maniement transférentiel dans sa relation à l'hostilité des patients, ce point étant l'un des principaux dans le travail auprès d'adolescents violents. Comment travailler avec un patient qui montre son hostilité «à ciel ouvert»? 


\section{À propos d'une clinique possible de l'adolescence: le travail sur la demande}

La clinique psychanalytique avec des adolescents en conflit avec la loi impose tout de suite une complication à la pratique: il s'agit de travailler la demande initiale et d'en faire une demande analytique, puisque ces jeunes trouvent dans l'acte une ressource principale pour gérer l'angoisse. Le travail de la demande exige un temps d'attente qui, parfois, ne peut être supporté par des sujets sans la précipitation de l'agir dans le cadre psychanalytique.

Dans ce contexte, le rapport à l'analyste peut représenter une menace, en tant que porte ouverte pour que ce qui est de l'ordre du traumatisme se réalise dans le cadre analytique. Ainsi, être avec l'analyste peut signifier la possibilité d'une relation à celui-ci. Cependant, si dans le rapport à l'Autre l'adolescent se sent livré à sa voracité, comment ne pas agir, comment ne pas se défendre du rapport transférentiel à l'analyste? Comment négocier les angoisses persécutrices mobilisées par la proximité du psychanalyste? Et, tenant compte des nombreux agirs de l'adolescent, quelle est la place de l'analyste? Quel est le sens de son travail?

Dans un premier temps, la souffrance doit orienter le sujet dans son engagement dans une analyse. Ensuite, la demande d'aide doit devenir une énigme pour le sujet. S’il était possible, dans les entretiens préliminaires, de séparer ces moments de la demande, il y en aurait deux. Le premier: une demande sous mandat pendant lequel le sujet reconnait ou est mené à reconnaître sa souffrance. Le deuxième: la transformation de la demande d'aide en une demande d'analyse, où le patient élabore une question sur son symptôme. Néanmoins, la clinique avec des adolescents, notamment avec ceux qui privilégient l'acte, semble avoir du mal à s'organiser ainsi en deux temps distincts.

Dans l'accueil des adolescents, l'analyste doit se pencher sur la détresse, sur la souffrance, reconnues ou non par le sujet, avant de se précipiter dans l'aide à la construction d'une question, c'est-à-dire, une demande d'analyse. Coutinho (2006) souligne que l'instauration d'un espace de parole, d'accueil et de construction d'un lien à l'adolescent doit être privilégiée et rappelle qu'il est essentiel, dans la conduite de l'analyse, d'accompagner l'adolescent dans le travail d'inscription de l'agir dans l'ordre de la parole.

Dans cette clinique, le pari est que le transfert constitue une tentative d'amener le sujet à modifier le rapport entre angoisse et agir, c'est-à-dire de les dissocier et de créer un espace de symbolisation entre les deux. Selon Pinel (2007), malgré leur charge destructrice, certains actes violents sont porteurs 
d'un désir de signification et parfois, le travail sur le cadre peut rendre possible le processus de symbolisation. Nous présenterons ci-dessous, dans une vignette clinique ${ }^{2}$, le cadre en tant que promoteur de la construction du lien dans le rapport entre l'analyste et l'adolescent.

La prise en charge psychothérapique d'orientation psychanalytique dont il sera question a eu lieu dans un espace de placement où l'adolescent se trouvait privé de liberté, au sein d'une institution sociale à Brasília, la capitale du Brésil. Cette thérapie a été menée par des étudiants en psychologie en stage clinique, supervisés par des enseignants de Cliniques-écoles de deux universités.

\section{Le cas Tiago $^{3}$ et le cadre en tant que promoteur du lien transferentiel}

Tiago avait 18 ans au début du suivi clinique effectué en institution de placement pour adolescents en conflit avec la loi, dans laquelle il se trouvait depuis environ un an. Le suivi hebdomadaire a duré 10 mois.

La famille de l'adolescent se composait d'une soeur de 25 ans et d'un frère de 21 ans. Jusqu'à ses huit ans, ses parents étaient mariés, dans une relation tumultueuse, et l'enfant a vécu d'innombrables scènes de violence entre eux. "Je voyais mon père saoul frappant ma mère». Ensuite son père est parti sans donner de nouvelles. Il est toutefois revenu pour visiter Tiago dans l'institution. Trois ans après le divorce, sa mère a trouvé un autre compagnon, leur relation a perduré jusqu'au début de la prise en charge clinique. Pour l'analyse du cas, nous désignerons la situation de violence domestique comme «scène 1 ».

Dans les séances, Tiago affirmait que la vie dans le crime «était pas mal» parce qu'elle lui procurait «de l'adrénaline, du danger». Il volait des voitures et des maisons, dans des attaques à main armée, et avec l'argent du butin, il allait au centre commercial s'acheter des vêtements de marque. À la question posée sur l'adrénaline, il répondait, tapant son avant-bras: «C’est de l'adrénaline dans les veines.» Il disait qu'au moment du vol, il pensait «Que fais-je si la police arrive? » et que face à cette possibilité, tout devenait encore plus excitant.

Un jour, coincé par la police, Tiago a été envoyé dans une institution fermée. Sa mère le visitait fréquemment et son père, après des années d'absence, était aussi venu lui rendre quelques visites. Tiago disait qu'il n’appréciait pas les visites de son père, mais qu'il les acceptait suivant la demande de sa mère et de sa sœur qui aimeraient qu'il reprenne sa relation avec lui. Tiago 
ne lui a jamais posé de question sur sa disparition qui a duré presque 10 ans, et ce, même s'il restait intrigué par les raisons de cet éloignement. Il ne s'est jamais autorisé à lui poser ce genre de question, attendant qu'il puisse lui en parler de lui-même.

Sa détention, que nous appellerons «scène 2 », s'est passée à la suite d'une situation transgressive, racontée comme suit: «Avec un ami, nous avons volé une voiture, mais nous ne nous sommes pas rendu compte qu'il y avait une dame dedans. Un gendarme s'est pointé et m'a tiré dessus plusieurs fois.» L'autre version de l'histoire est la suivante: «Je ne voulais plus voler, je n'y trouvais plus de désir. Mais on passait à côté d'une voiture et mon ami, qui voulait bien voler, a menacé une dame dans un véhicule.» Tiago a assumé le rôle qui lui était destiné: il s'est assis à côté de la victime, l'arme sur sa tête, tandis que son ami conduisait la voiture. La police arrive, il y a des échanges de tirs, son ami s'enfuit, mais Tiago est capturé. Quand il a parlé de son rôle dans les vols, il a dit qu'il pointait l'arme contre la tête de la victime, en la menaçant. Il semblait avoir du plaisir en exerçant du pouvoir sur ses victimes.

Le cas clinique a été marqué par ces deux scènes paradigmatiques dans l'histoire de Tiago et dans la trame d'un adolescent en conflit avec la loi. Les deux scènes possèdent des éléments qui constituent l'axe principal dans l'analyse du cas: la scène 1 concerne une situation traumatique, soit la violence et l'abandon du père. La scène 2 illustre la situation menant à son placement. Dans la scène 1, Tiago assiste avec passivité à la violence du père contre la mère et reste passif face à l'abandon paternel. Dans la scène 2 , l'adolescent quitte la position passive et assume la place de l'agresseur.

\section{Demande et transfert : actualisation des scènes}

Avec Tiago, le travail de construction d'une demande a subi deux complications. D’abord, Tiago ne semblait pas être en contact avec sa souffrance. Il ne pouvait même pas entrevoir ce qui était du registre de sa détresse, soulignant l'aisance avec laquelle il se sortait des situations difficiles et se vantant du fait de ne pas être affecté par les situations problématiques de sa vie. Il semble que le fait d'être en contact avec sa détresse le mettrait dans une position de passivité et de vulnérabilité, ce dont il se défendait.

Face à une situation de douleur qui ne peut être symbolisée et devient souffrance psychique, le sujet peut fonctionner sur la base de la neutralisation affective et sous le registre de l'acte et de l'agir adressé à l'autre. Ainsi, la clinique du passage à l'acte porte un message (non verbal) adressé à l'autre, comme une tentative de communiquer, de partager et de soumettre l'autre 
à des situations traumatiques que le sujet ne peut symboliser intérieurement. Dans ce cas, l'analyste est confronté au manque de sens et de liaison. De ce point de vue, la problématique est celle de l'agir comme tentative de symbolisation, passant par l'acte et non par le refoulement. L'autre, comme objet de projection, devient également objet de support pour une subjectivation qui ne s'est pas intériorisée. D'autre part, Marty (2003) souligne que l'acte violent à l'adolescence peut constituer une voie de quête de plaisir, essentiellement narcissique, afin de vider l'appareil psychique des exigences de travail de liaison et de représentation des effets des traumatismes. L'agir devient ainsi un instrument de lutte contre la menace de désintégration et de destruction du sujet.

Soutenue par un rapport transférentiel qui se consolidait, l'analyste a été capable de montrer à Tiago qu'en réalité il ne s'est pas toujours bien sorti des situations difficiles. Celles-ci lui ont coûté cher et sont devenues depuis un poids à porter. Toutefois, Tiago résistait à se confronter à sa souffrance. De ce fait, l'analyste a changé sa stratégie et a décidé d'utiliser la dimension imaginaire dans laquelle il semblait être pris. Elle a alors commencé à réagir aux récits de Tiago par des expressions faciales, comme si elle était étonnée par les moments difficiles qu'il a vécus. Sans rien dire, il la regardait de façon énigmatique. C'est alors par le corps de l'analyste, par ses expressions faciales que la communication avec Tiago a acquis du sens. Au tout début de la constitution psychique, Freud (1895) affirme que le bébé, plongé dans la détresse originelle, appelle l'autre au moyen de pleurs et de la motricité incontrôlée. Les actes montrent le manque de confort du bébé, mais la mère suppose que dans l'infans existe un sujet de désir et l'interprète. L'analyste a soutenu une fonction semblable à la fonction maternelle dans ces épisodes de la thérapie de Tiago.

La deuxième complication dans la construction de la demande, dans le cadre clinique, s'est présentée quand Tiago a essayé de tester l'analyste: en tant qu'adolescent abandonné par le père et devenu marginal, avec ses règles à lui, pourrait-il avoir confiance en quelqu'un? Au début, il a précisé: «Je ne parle que si vous me posez des questions.» Et ensuite: «J'ai appris à être comme ça dans le monde du crime, à ne faire confiance à personne.» Les professionnels qui entouraient Tiago étaient des représentants de l'État. Cependant, l'analyste lui offrait une écoute libre de toute censure et sans aucun lien à l'État. Une place pour le transfert était indispensable pour la construction, avec l'analyste, d'un rapport différent des autres. L'analyste n'appartenait pas au monde du crime, elle n'était pas une fonctionnaire de 
la justice: en fin de compte, que pourrait-elle lui demander? Comment faire confiance à quelqu'un qui ne faisait qu'écouter sa souffrance, une émotion difficile qu'il ne se permettait pas de rencontrer? Peu à peu la construction d'un rapport transférentiel a permis que ce jeune puisse se confier à son analyste. Pour cela, il a fallu qu'elle ne manifeste aucune expression corporelle ou interprétation signifiant un jugement.

Il est important d'expliciter les défis du cadre, car le traitement était réalisé dans l'unité de santé de l'institution, chaque jour dans une salle différente. L'agent de sécurité restait à la porte de la salle et parfois il insistait sur le besoin de laisser la porte entrouverte, comme un signe de protection de l'analyste par rapport à Tiago. En revanche, l'analyste insistait sur la question de la confidentialité et gardait la porte fermée dans une tentative d'assurer les espaces du dedans et du dehors dans le cadre psychothérapeutique. Même avec quelques modifications dans l'espace physique, telles que le changement de salle, l'écoute de l'analyste resterait stable.

Pour Tiago, le cadre était menaçant. Il était toujours prêt à se défendre et au début, son regard et son attention étaient centrés sur l'analyste. Celle-ci a alors décidé de redéfinir la notion de cadre, en expliquant qu'elle n'avait pas de contrôle sur le choix de la salle, c'est-à-dire qu'elle n'avait pas le pouvoir de décision sur cet espace. Elle lui montrait ainsi que l'espace physique ne leur appartenait pas, contrairement au registre de la parole. Celui-ci constituait l'espace de travail indépendamment du lieu où se passaient les séances.

Un jour, un fait curieux est advenu. Les adolescents arrivaient sur le lieu du soin, tête basse et mains dans le dos, accompagnés par l'agent. Ils attendaient l'analyste dans une petite salle d'attente, dans l'infirmerie. De retour des vacances de Noël, Tiago n'a pas attendu l'analyste et est allé la chercher dans les différentes salles. Celle-ci a entendu l'agent lui dire qu'il ne pouvait pas savoir où elle se trouvait. Tiago a insisté, avec succès. Il est entré dans la salle, il a fermé la porte et s'est assis. La salle elle-même n'était pas l'important, il s'agissait à contrario de s'assurer de la disponibilité de l'analyste.

Quant au transfert, pendant presque tout le suivi thérapeutique, Tiago a adopté une posture de confrontation où il regardait constamment l'analyste en observant tous ses mouvements. Celle-ci se sentait enfermée, coincée et angoissée. Elle savait bien, toutefois, que sa posture devait éviter de représenter une menace pour Tiago, car il semblait méfiant et prêt à l'attaque si l'analyste se moulait à ses projections en devenant par exemple rude ou en le renvoyant. L'agressivité pourrait alors devenir sa façon de se défendre aux moments où il revivrait le traumatisme de l'abandon et de la violence. 
Dans cette posture de confrontation, Tiago semblait provoquer l'analyste, comme il provoquait d'autres professionnels et les adultes qu'il rencontrait, suivant des fantasmes potentiels d'abandon ou de persécution. Il semblait demander à l'analyste de le renvoyer ou de montrer sa peur, comme si, face à lui, elle était sa victime. L'analyste n'a pas cédé à cette demande. Bien au contraire, elle a soutenu la position d'un Autre accueillant mais ferme, là où les adultes de référence avaient échoué.

Le travail d'analyse est un parti pris pour la parole, afin de singulariser la constitution d'une demande analytique, dans laquelle l'analyste témoigne du désir du patient adressé à un Autre; la figure de l'analyste est susceptible d'incarner cet Autre dans le transfert. Il n'est pas un «petit autre» qui reconnaît et répond à ses demandes (de reconnaissance et d'amour), d'où la référence à sa fermeté.

En refusant d'occuper la position de reconnaissance de la demande première qui lui est adressée, en acceptant d'écouter le sujet dans sa détresse, l'analyste soutient une fonction élaborative et fait preuve de l'éthique de la psychanalyse. De fait, ce n'est pas en répondant à cette demande et en muselant ainsi le désir du sujet qu'une demande d'analyse pourra se constituer.

Il a fallu supporter ce que Tiago a adressé à l'analyste, lui permettant de se mettre à cette place, sans néanmoins répondre à sa demande. Dans un premier moment, il demandait d'être «re-chassé», et par là il s'engageait dans le traitement. En revanche, l'analyste ne pouvait pas répondre à l'agression par une interprétation destructive ou par des mots agressifs. En 1912, dans La Dynamique du Transfert, Freud affirme que le transfert se joue dans un paradoxe: dans le même temps qu'il est la résistance la plus puissante au traitement, il est également une condition à la réussite du travail psychanalytique. Le transfert est divisé entre deux versants, positif et négatif: le premier est lié aux sentiments amicaux et affectueux envers l'analyste et le deuxième réfère à une position hostile et peut se présenter, par exemple, par une certaine agressivité adressée à l'analyste ou par la dévalorisation de son rôle. Tous deux s'appuient sur des pulsions érotiques primitives et refoulées, manifestées par le moyen de la dynamique transférentielle.

Plus d'une fois avec Tiago, l'analyste a souhaité ponctuer de façon plus explicite et ferme, tout en réalisant combien l'adolescent était désemparé et souffrant face à cela. Cependant, elle a pu reconnaître que cette voie risquait de rendre à Tiago l'agressivité qu'il lui adressait, et que ce serait comme lui dire: «Pour qui te prends-tu pour m'affronter comme ça? Tu n'es qu'un pauvre type!» Il a fallu que l'analyste se mette à l'écoute de son propre 
inconscient pour repositionner son désir d'écouter le patient et apprivoiser le «cheval furieux» que représentaient les réactions transférentielles négatives. L'analyse propose justement de soutenir le désir qui effleure l'écoute pour le rendre plus conscient à l'analysant, en tant qu'objet orientant le travail d'analyse.

De ce fait, en lui offrant son écoute, l'analyste a contribué à ce qu'une demande d'analyse puisse apparaître, mobilisée par la scène 2. Tiago a raconté à l'analyste, à plusieurs reprises, la scène du vol. Cependant, à chaque récit, un élément nouveau était ajouté et ainsi, la scène n'était pas toujours la même. Il a d'abord dit qu'il ne savait pas qu'il y avait une dame dans la voiture. Puis il a dit qu'il savait qu'elle y était et qu'il «a fini»par la prendre en otage. Finalement, il a affirmé qu'avec son ami, ils voulaient voler la voiture et séquestrer la victime. En racontant la scène 2 à l'analyste et en ajoutant des détails apparemment effrayants, Tiago testait son analyste: comme s'il vérifiait qu'elle le recevrait encore, suite au récit de ce qu'il a été capable de faire. Almeida et Atallah (2008) soulignent que la remémoration (erinnerung) est orientée vers le non représentable, puisque la mémoire est pleine de lacunes impossibles à combler par l'univers symbolique du sujet; en conséquence, la parole du sujet est toujours inachevée.

La confiance étant établie dans le transfert, il a été possible d'accéder, d'élargir et de relativiser le monde imaginaire de Tiago, et d'ouvrir, minimalement, une porte au monde fantasmatique. La recherche de la signification de l'agir constitue une des orientations thérapeutiques avec ces adolescents. Nous pensons qu'avec Tiago, il y a eu début d'une symbolisation dans la construction d'un espace psychique fantasmatique différent de l'agir, dont l'articulation avec les questions conflictuelles inconscientes a été retrouvée plus tard.

Tiago a utilisé l'agir dans l'espace thérapeutique, dans une tentative de réactualiser la détresse ou de mettre l'analyste en position de victime: «Ou elle, ou lui». Or, l'analyste a la possibilité d'outrepasser ces demandes initiales. L'analyste paie « [...] avec sa personne, dans la mesure où, en tout état de cause, il la prête comme support aux phénomènes singuliers révélés par l'analyse dans le transfert» (Lacan, 1958, p. 593). Il doit maintenir sa non réponse aux différentes modalités de la demande première, tout en encourageant l'analysant à utiliser la parole. Une partie du maniement transférentiel se fait par l'invitation à la parole face à l'acte.

Nous nous référerons ci-après à l'angoisse et au terme erwartung — attente - utilisé par Freud (1917), pour penser un parcours clinique visant la reprise de la parole. 


\section{Erwartung - un parcours clinique pour l'élaboration psychique}

L'éveil de l'angoisse ne se réalise pas par la présence de l'Autre, mais par la confrontation au désir de l'Autre, faisant apparaître la détresse originelle (hilflosigkeit). Lacan (1960-61) reprend un concept freudien (1917) pouvant s'avérer important dans la cure: celui de l'attente, ou erwartung. De fait, l'importance de l'angoisse dans le processus psychanalytique est liée à l'apport, par l'analyste, de la fonction de l'attente. Dans la détresse, le sujet est troublé, il ne réussit pas à dépasser cette situation autrement qu'en cherchant à fuir. Mais entre la détresse et la fuite, une autre possibilité tient à l'angoisse, à son caractère d'erwartung — attente.

L'angoisse est un signe qui met le sujet en état d'attente face au danger futur. Elle autorise quelque chose de l'ordre de l'attente permettant non seulement à la détresse d'apparaître, mais également à l'élaboration psychique de s'amorcer. Ainsi, l'angoisse comprend un caractère productif, car l'attente de quelque chose présuppose un temps de compréhension et d'élaboration (Chediak, 2007). Tiago ne démontrait pas l'affect d'angoisse aux premiers moments de son discours ou de ses récits. Il ne se permettait pas de ressentir l'angoisse et s'empressait de la contrer par ses actes, en refusant le temps de l'attente qui pourtant permet l'élaboration psychique. Au cours des séances, assumant une posture corporelle de confrontation, il regardait fixement l'analyste en lui disant: «Je ne parle que si vous me posez des questions.»

Au début de la thérapie, l'angoisse était toutefois présente chez l'analyste qui incitait Tiago à parler. Celui-ci parlait, répondait à toutes les questions de l'analyste en la coinçant en permanence avec son regard. Cela s'effectuait tranquillement, apparemment en toute sérénité. L'analyste était dans une posture inconfortable et se demandait où se jouait l'angoisse de cet adolescent. Peu à peu, elle a adopté une posture plus décontractée $e^{4}$ en lui offrant, par sa position physique, un temps d'attente permettant une élaboration, sans passage direct à la parole pleine ou à l'acte.

Suite à cette nouvelle position, Tiago a semblé ébranlé et a repris spontanément plusieurs questions qu'elle lui avait posées auparavant. Angoissé par le fait de répéter les mêmes réponses, bien que gêné par l'allure décontractée de l'analyste, il a commencé à raconter son quotidien dans l'institution en révélant des secrets et ses projets de fuite. Il a raconté la scène 1 de façon répétée, démontrant éventuellement un certain niveau d'élaboration.

L'angoisse a été considérée comme le signe d'une demande d'aide pour supporter la détresse, car l'appareil psychique n'en avait pas la possibilité. Elle venait signaler le besoin d'un travail d'élaboration. Il ne suffisait pas 
de raconter la scène, il fallait la revisiter et la revoir. L'analyste relevait les remodelages de la scène dans ces moindres différences. Elle ne faisait pas de remarques sur les divergences dans les récits; bien au contraire, elle les écoutait et poursuivait ses interventions. Tout cela s'est passé dans le temps de suspension de l'acte, le temps de l'attente.

Les premières fois que l'adolescent a raconté la scène 2, il abordait rapidement son rôle par rapport aux victimes et à l'apparition de la police. Concernant la victime, d'abord, il ne voulait que son argent. Ensuite, il a affirmé que c'était lui qui la coinçait. Puis il a dit que c'était à lui de «garder» les victimes. Enfin, il s'est reconnu comme une figure menaçante, car il menaçait ses victimes de mort et il voulait voir la peur sur leur visage. En ce qui concerne l'agression du gendarme - un représentant de la loi —, au début elle était considérée comme un simple malheur, mais quand Tiago a pu mentionner "la sensation d'adrénaline», un nouveau champ s'est ouvert dans son discours. D'une certaine façon, il attendait l'arrivée du gendarme et avec son apparition, il ne s'est plus occupé de la souffrance de la victime; pour lui, il est devenu plus important de tirer sur le policier. Puis, une fois de plus, «le malheur»: Tiago a été atteint. L'analyste centrait ses interventions sur ce «quelque chose de nouveau» dans son discours, en cherchant à élargir chez le jeune la dimension symbolique par la parole.

Aulagnier (1995, p. 6) affirme que «le sujet est l'affect-angoisse; il le vit de façon globale, et cette imprégnation, cette capture du moi qui se dissout dans l'angoisse, est effectivement ce qui empêche la médiation par la parole». Au cours des dernières séances, Tiago a parlé de son plaisir lié à la souffrance de l'autre et de sa haine contre son père. Il a quand même exprimé certains sentiments par rapport à ce qui s'est passé avec son père, mentionné au début du traitement comme «celui qui ne représente rien dans ma vie». Ainsi, mettre en paroles signifie transformer en communicable ce qui a été vécu au niveau du corps, tout en prenant, en même temps, une distance infime par rapport au vécu affectif. Pour Aulagnier (1995), ceci montrerait que le moi a déjà acquis une certaine maîtrise et une objectivité face à un affect.

Freud (1930 [1929]) propose les notions de sublimation et de fantaisie en tant que processus psychiques pour échapper à la souffrance, en déplaçant la libido pour éluder la frustration du monde extérieur. «[...] Cette procédure montre déjà clairement une intention de nous rendre indépendants du monde extérieur par la quête de satisfaction en processus psychiques internes» (p. 88). Ceci constituerait le mouvement de l'analyse, non 
seulement dans le sens sublimatoire, mais par la quête de satisfaction dans l'élaboration, soit un processus psychique interne. Le sujet n'a pas besoin d'un acte qui puisse court-circuiter le processus de symbolisation, puisqu'au-delà de l'acte, il accède à d'autres ressources. "Comme le commerçant diligent qui évite de placer tout son argent dans une seule affaire, peut-être le savoir populaire puisse nous conseiller à ne pas chercher toutes nos satisfactions dans une aspiration unique» (Freud, 1930 [1929], p. 91). Pour Tiago, ne plus miser entièrement sur la criminalité demanderait un recours au symbolique lui permettant de s'affranchir de l'emprise de l'omniprésence maternelle. Autrement, l'agir surgit dans le monde extérieur, à partir de l'impossible élaboration de l'angoisse par le moyen d'un processus interne.

Les demandes de Tiago sont devenues plus complexes dans la mesure où ses fantaisies se présentent désormais en haut-relief, avec des références au transfert (l'analyste s'abstenant de répondre à partir de la position demandée) et un travail relatif au champ de la parole. On peut considérer alors que l'analyse a permis une certaine élaboration psychique de la part de Tiago car quelques associations et des liens ont été produits, que se soit dans l'exposition d'affects (comme la haine contre son père et la jouissance de ses propres actes criminels) ou dans son rapport à son histoire de vie.

En fait, bien que l'acte se donne à l'interprétation psychanalytique et permette d'entrevoir les parcours du désir adolescent, il a aussi la capacité de se taire. Tel que mentionné précédemment, nous faisons référence ici à des parcours précaires de symbolisation. En ce sens, Sousa (2000) affirme que l'acte violent est une rupture avec le dialogue et ne contribue en rien à la réparation du lien social, car il empêche la circulation de la parole. De même, Melman (2003, p. 69) affirme que «la violence surgit au moment où les mots deviennent inefficaces». Quand le sujet n'est plus reconnu en tant que sujet, quand sa parole n'est pas la voie pour l'obtention de cette reconnaissance, advient la violence. Celle-ci peut être comprise comme le déclin du pouvoir de la parole: l'autre n'étant pas reconnu comme semblable - il est alors impossible de mettre les paroles en circulation. L'acte violent signale une impasse dans le maintien des liens sociaux et en conséquence, il rend muet le sujet.

C'est dans l'impasse de l'acte, dans la faille de la symbolisation et lorsque les paroles se taisent, que la clinique psychanalytique peut montrer son pouvoir. Freud nous a parlé du pouvoir de la parole et Lacan a révélé le caractère apaisant du symbolique. La clinique nous convoque à accorder du poids à la parole et à impliquer le sujet dans son discours et dans son désir. 


\section{Les niveaux du transfert}

L'analyse du transfert, dans ce cas clinique, peut se réaliser en deux niveaux. Le premier, déjà travaillé au cours des paragraphes précédents, se situe dans le contexte de la relation analytique entre Tiago et l'analyste. Même apparemment réceptif à la proposition d'analyse, le jeune homme assumait une position de confrontation et de menace. Au cours de la thérapie, l'angoisse est apparue du côté de l'analyste, et du côté de l'adolescent se révélait le passage à l'acte.

Deux réponses étaient possibles de la part de l'analyste, confronté au transfert négatif: se positionner en tant que victime coincée face à l'intimidation de Tiago ou lui répondre, de façon également agressive, jusqu’à l'expulser de l'analyse. Cependant, l'analyste a réussi à manier le transfert sans tomber dans le jeu de l'analysant. Avec une certaine tranquillité, faisant semblant d'être insouciante, l'analyste a cherché une troisième position pour que l'angoisse puisse émerger du côté de Tiago, ce qui a permis le temps d'attente et d'élaboration psychique des scènes.

Le deuxième niveau est celui du transfert institutionnel, celui-ci étant essentiel pour dépasser nettement le premier. Évidemment, au début de la thérapie, Tiago s'adressait à l'analyste en lui disant qu'il avait changé et qu'il ne voulait plus mener sa vie dans le monde du crime. En fait, il ne s'adressait pas vraiment à l'analyste mais à une personne représentant l'institution. Le cadre analytique n'était pas, au début, différencié du cadre institutionnel. Il fallait donc que l'analyste soit attentive à cet aspect car celui-ci pouvait avoir une influence sur le processus analytique.

Dans le cas de Tiago, l'institution a rendu impossible, d'une certaine façon, la continuité du travail. En effet, au cours des derniers mois, en raison de problèmes dans l'institution, l'analyste a été souvent empêchée de le recevoir. La gestion de l'institution expliquait la réduction du nombre de séances par la réduction du nombre d'agents de sécurité, l'absence d'espace physique pour les soins, ou l'impossibilité de le faire quitter l'espace de récréation où des adolescentes se mettaient au soleil. Quand il ne venait pas à la séance, dans la séance suivante l'analyste l'informait des raisons alléguées. Les absences de Tiago sont devenues fréquentes jusqu'au moment où il ne s'est plus rendu aux séances. L'institution avait envahi l'espace du cadre, établi malgré les conditions plutôt adverses.

Il n'y avait que deux possibilités de rencontrer Tiago: soit dans l'unité de santé (où se passaient les prises en charge), ou alors par l'intermédiaire de l'éducatrice qui pouvait fixer un rendez-vous en dehors de l'horaire prévu. 
La première a été rendue impossible par l'institution, de telle façon que Tiago a refusé de venir pendant quatre semaines. La deuxième, par l'intermédiaire de la professionnelle, s'est avérée essentielle. Cependant, malgré sa participation, elle s'objectait depuis le début à la thérapie, arguant qu'il s'agissait d'une perte de temps puisque Tiago était un cas impossible. Pour elle, l'analyste devrait offrir son écoute à ceux qui voulaient vraiment changer, ce qui n'était sans doute pas le cas de Tiago. Pendant un mois, l'analyste a demandé un rendez-vous avec Tiago, mais la professionnelle responsable ne le fixait jamais, en insistant pour que l'analyste reçoive d'autres adolescents. Après six semaines, l'analyste a laissé tomber. Les croyances et l'attitude de la professionnelle ainsi que l'organisation de l'institution ont envahi le cadre de l'analyse, rendant sa continuité impossible.

Suite à cette expérience, certains aspects peuvent être soulignés aussi bien en ce qui concerne le maniement transférentiel que le cadre. À partir d'une certaine «insouciance» par rapport à l'environnement institutionnel, il a été possible de vérifier que le cadre ne constitue pas seulement une toile de projection de l'analysant, mais aussi un espace pour le travail de l'analyste. N'importe quel cadre analytique peut permettre la construction d'un nouveau registre psychique pour le sujet, pouvant se constituer en un lieu où ses actes sont marqués par de nouvelles significations. Cependant, la position de la professionnelle responsable de Tiago a montré que l'institution, qui soutient le discours de la réhabilitation et de la réintégration sociale de tous les adolescents, peut se présenter en fait comme une entrave cruelle à la construction d'un cadre psychothérapeutique. Finalement, les interventions de cette professionnelle se sont orientées vers le choix des adolescents qui mériteraient d'être suivis en analyse, c'est-à-dire ceux qui, selon son évaluation, pourraient profiter de ce que l'institution leur offrait.

À partir de cette perspective, la prise en charge psychothérapique risquait de devenir une monnaie d'échange entre l'adolescent et l'institution.

\section{Considérations finales}

La clinique psychanalytique avec des adolescents renvoie le sujet à une question singulière, à une énigme, non pas pour y répondre ou pour l'étouffer, mais pour impliquer le sujet dans son désir et dans ses symptômes. L'adolescent pourra peut-être se désespérer face au leurre de la demande, car même si celle-ci se démultiplie en plusieurs demandes qui se chevauchent, le manque de réponse de l'analyste montre un désir insatiable à l'horizon (Alberti, 2009). Pour dévoiler le mystère caché à la base de son désir, c'est le 
désir de l'analyste qui autorise l'adolescent, au moment où il l'accueille et écoute sa détresse, en signalant l'insatisfaction du désir et l'impossibilité du rapport sexuel.

Lesourd (2004) propose que dans l'analyse d'adolescents, en particulier ceux qui sont marqués par des comportements de transgression, l'analyste travaille dans le sens de permettre au sujet d'assumer la responsabilité de ses actes. Il distingue l'acte et l'agir. L'agir constitue la voie empruntée face à l'angoisse, pour être sûr de son existence - une certitude narcissique, l'aliénant encore plus dans l'Autre. D'autre part, nous avons vu que l'acte (comme dans Totem et tabou) marque l'apparition du sujet et constitue une énonciation subjective. Le passage de l'un à l'autre demande l'inscription de l'agir dans l'ordre de la parole. La dimension de l'acte concerne la reconnaissance du sujet en tant qu'auteur, au niveau de l'inconscient. Dans la dimension consciente, le délinquant sait sans doute qu'il en est l'auteur, mais ce que l'acte lui apporte, au-delà du plan conscient, est la marque de son désir.

Néanmoins, le travail consistant à renvoyer le sujet au champ du symbolique est particulièrement difficile dans la clinique où l'agir est prédominant. L'agir réapparait dans le processus psychanalytique, de façon privilégiée, comme une impulsion de rupture du rapport transférentiel, comme une marque du transfert négatif (Amparo, Gusmão et Vilas Boas, 2012). L'angoisse du processus adolescent, en particulier chez les adolescents en conflit avec la loi, les renvoie à une difficulté à la symboliser. Ainsi, l'urgence de l'agir peut indiquer une difficulté dans le maniement de la pulsion: autrement dit, une restriction du recours symbolique (Balier, 1996).

L'hypothèse de Marty (2010) selon laquelle le recours à l'acte est une déviation du conflit intérieur dans l'obtention de plaisir montre que, dans le travail analytique, il faut chercher une autre source de plaisir: l'élaboration psychique, dans l'association d'affects à des représentions et dans la liaison de représentations actuelles à des traces mnésiques. À l'adolescence, ce processus est très difficile, car se rappeler nécessite de faire place au lien incestueux et parricide de l'Edipe. «L'acte prend la place d'une pensée et, plus précisément, d'un souvenir qui n'a pas pu être élaboré» (p. 50). Le fantasme incestueux est traduit dans la violence: dans l'impossibilité de maitriser l'angoisse, l'acte se présente comme le seul recours.

L'ordre symbolique, depuis la dialectique spéculaire, porte une fonction d'apaisement. Après tout, la loi, l'interdiction instaurée par le père dans le registre du langage, intervient dans la relation spéculaire introduisant le rythme symbolique (Lacan, 1953-54). Non par hasard, les adolescents pris 
dans une position narcissique se présentent par le moyen d'une violence inquiétante. C'est l'instauration de la loi qui, de façon paradoxale, intègre le sujet dans la sexualité, car la reconnaissance de la position sexuelle du sujet est liée au système symbolique (Lacan, 1953). Ne serions-nous pas en train de parler d'une errance, provenant de la rencontre avec le pubertaire, propre à l'adolescence?

Récupérer le sujet par le moyen de la dimension de la parole est un défi, car la violence la muselle. L'analyste travaille, via la potentialisation de la parole, pour que le sujet puisse se l'approprier et reconnaître l'autre comme son semblable. Il s'agit d'impliquer le sujet dans ce qu'il dit, dans la mesure où la parole a un statut de vérité. Ce mouvement constitue ce que Lacan (1953-54) a désigné comme réintégration symbolique de l'histoire du sujet, qui cherche l'appropriation, par le sujet, de son histoire, réinventée et réécrite tout au long du travail psychanalytique.

Dans le cas de Tiago, l'analyste, s'éloignant de la demande (imaginaire) de reconnaissance et de rejet exprimée par le comportement narcissique et hostile de l'adolescent, l'a aidé dans la construction d'une demande d'analyse qui l'a renvoyé à l'énigme de son désir. Ainsi, un travail d'élaboration psychique a été possible, grâce au maniement du rapport transférentiel, même dans les conditions adverses imposées au cadre clinique par l'institution.

Le traitement de Tiago n'a pas eu de continuité, ce qui a provoqué un sentiment de frustration chez l'analyste et les superviseurs de sa pratique clinique. Néanmoins, l'expérience apportée par ce cas clinique a renouvelé notre désir de mener des interventions psychanalytiques avec des adolescents en conflit avec la loi et en situation de placement, pariant sur les effets de l'écoute et de la circulation de la parole en tant que ressources privilégiées pour assumer le désir, là où très souvent il n'y a même pas de supposition de sujet.

Laís Macêdo Vilas Boas

Deise Matos do Amparo

Sandra Francesca Conte de Almeida

Katia Cristina Tarouquella R. Brasil ktarouquella@gmail.com 


\section{Notes}

1. Recherche réalisée avec le soutien financier du Conseil National de Développement Scientifique et Technologique - CNPq. Traduction du portugais en français par Inesita Machado, avec révision technique de Sandra Francesca Conte de Almeida et Sophie Gilbert.

2. L'étude de cas clinique concernant cette vignette a été développée de façon plus approfondie dans la Dissertação de Mestrado (Master), à l'Université de Brasília, par Villas Boas, 2013.

3. Il s'agit bien sûr d'un prénom fictif.

4. Le terme « décontractée » réfère à un choix de posture destiné à introduire une inconnue dans le transfert et ébranler la position antérieure de l'analyste, c'est-à-dire, l'angoisse.

\section{Références}

Alberti, S. (2009). Esse sujeito adolescente. Rio de Janeiro: Rios Ambiciosos.

Almeida, L. P., De, Atallah, R. M. F. (2008). O conceito de repetição e sua importância para a teoria psicanalítica. Ágora (Rio J.), 11 (2), 203-218.

Amparo, D.M., Gusmão, M.M., Vilas Boas, L.M. (2012). O agir violento na adolescência: simbolização e dispositivos clínicos. Dans D. M. Amparo, S. F. C. de Almeida, K. T. Brasil, M. I. Conceição, et F. Marty (dir.), Adolescência e Violência: Intervenções e Estudos Clínicos, Psicossociais e Educacionais (p. 79-91). Brasília: UnB/Liberlivros.

Arnaux, D. J. (2003). Le psychanalyste, l'éthique et l'institution. Dans F. Marty, P. Gutton, P. Givre, (dir.), Le fait accompli dans la psychothérapie de l'adolescent (p. 195-208). Rouen, France: Publications de l'universite de Rouen.

Aulagnier, P. (1995). Angústia e Identificação. Percurso, 14 (1), 5-14.

Balier, C. (1996). Psychanalyse des comportements sexuels violents. Paris: Presses universitaires de France.

Chediak, G.F. (2007). Sobre a Angústia: um ensaio psicanalítico. Dissertação de Mestrado, Instituto de Psicologia, Universidade de Brasília.

Coutinho, L.C. (2006). Pensando sobre as especificidades da clínica psicanalítica com adolescentes, Jornal de Psicopatologia Fundamental, 6 (2), 44-55.

Freud, S. (1896). Projeto para uma Psicologia Científica. Rio de Janeiro: Imago Edição Standard Brasileira das Obras Psicológicas Completas de Sigmund Freud, vol. I, 2006.

Freud, S. (1905 [1904]). Sobre a Psicoterapia. Rio de Janeiro: Imago Edição Standard Brasileira das Obras Psicológicas Completas de Sigmund Freud, vol. VII, 2006.

Freud, S. (1912). A Dinâmica da Transferência. Rio de Janeiro: Imago Edição Standard Brasileira das Obras Psicológicas Completas de Sigmund Freud, vol. XII, 2006.

Freud, S. (1913). Sobre o Início do Tratamento. Rio de Janeiro: Imago Edição Standard Brasileira das Obras Psicológicas Completas de Sigmund Freud, vol. XII, 2006.

Freud, S. (1914). Recordar, repetir e elaborar. Rio de Janeiro: Imago Edição Standard Brasileira das Obras Psicológicas Completas de Sigmund Freud, vol. XII, 2006.

Freud, S. (1916). Conferências Introdutórias sobre Psicanálise, Conferência XXV: A Ansiedade. Rio de Janeiro: Imago Edição Standard Brasileira das Obras Psicológicas Completas de Sigmund Freud, vol. XVI, 2006.

Freud, S. (1926). A Questão da Análise Leiga. Rio de Janeiro: Imago Edição Standard Brasileira das Obras Psicológicas Completas de Sigmund Freud, vol. XX, 2006.

Freud, S. (1930 [1929]). O Mal-estar na Civilização. Rio de Janeiro: Imago Edição Standard Brasileira das Obras Psicológicas Completas de Sigmund Freud, vol. XXI, 2006.

Lesourd, S. (2004). A construção adolescente do laço social. Petrópolis, Brésil: Vozes. 
Lacan, J.J. (1953). Função e campo da fala e da linguagem em psicanálise. Escritos. Rio de Janeiro: Jorge Zahar.

Lacan J. J (1953-54). O Seminário, livro 1: Os escritos técnicos de Freud. Rio de Janeiro: Jorge Zahar.

Lacan, J. J. (1958). A direção do tratamento e os princípios de seu poder, Escritos. Rio de Janeiro: Jorge Zahar.

Lacan, J. J., (1958). De uma questão preliminar a todo tratamento possível, Escritos. Rio de Janeiro: Jorge Zahar.

Lacan, J. J. (1960-61). O Seminário, livro 8: A transferência. Rio de Janeiro, Jorge Zahar.

Lins, M. I. A. (2006). Consultas terapêuticas: uma pratica clinica de D. Winnicott. São Paulo: Casa do Psicólogo.

Marty, F. (2010). Violências e passagem ao ato homicida na adolescência. Dans D. M. Amparo, S. F. C. de Almeida, K. T. Brasil et F. Marty (dir.), Adolescência e Violência: Teorias e Práticas nos campos Clínico, educacional e jurídico (p. 45-66). Brasília: UnB/Liberlivros.

Melman, C. (2003). O homem sem gravidade: gozar a qualquer preço. Rio de Janeiro: Companhia de Freud.

Pinel. J. P. (2007). Le traitement institutionnel des adolescents violents, Revue de psychothérapie psychanalytique de groupe. 1 (48), 109-122.

Quinet, A. (2002). As 4+1 condições da análise. Rio de Janeiro: Jorge Zahar.

Rocha, F. J. B. (2011). Entrevistas Preliminares em Psicanálise. São Paulo: Casa do Psicólogo.

Roussillon, R. (1995). Logiques et archéologiques du cadre psychanalytique. Paris: Presses universitaires de France.

Roussillon, R. (1999). Les enjeux de la symbolisation à l'adolescence. Adolescence, Monographie, 7-23.

Sousa, E.L.A. (2000). O silêncio da violência. Revista da Associação Psicanalítica de Porto Alegre. 19, 43-53.

Vilas Boas, L. M. (2013). A clínica do adolescente: vicissitudes da angústia e da transferência no agir violento. Dissertação de Mestrado. Instituto de Psicologia, Universidade de Brasília, Brasília-DF. 